\title{
Editorial
}

Theme: Nanoparticles in Vaccine Delivery

Guest Editor: Aliasger Salem

\section{Nanoparticles in Vaccine Delivery}

\author{
Aliasger K. Salem ${ }^{1,2}$
}

Received 9 January 2015; accepted 12 January 2015; published online 23 January 2015

Vaccines have been responsible for the effective control or elimination of many potentially fatal diseases. However, many other diseases such as cancers and those caused by intracellular pathogens still lack effective prophylactic or therapeutic vaccines. Furthermore, in developing countries, there is a need for vaccine formulations with stable shelf lives that eliminate or limit the number of boosts that are needed. With an increasing understanding of the immune system, much research has been focused on improving the current approach toward vaccine development in these areas. Nano and microparticle-based delivery systems have the potential to enhance the duration of antigen presence (depot formation), enhance dendritic cell (DC)-mediated antigen uptake, direct the stimulation of DCs, and promote cross-presentation. Nanoparticles also offer the potential to protect antigens and adjuvant from premature enzymatic and proteolytic degradation. Nanoparticle delivery systems offer the added strength of multi-component loading which is of considerable significance particularly in immunotherapy where simultaneous delivery of antigens, immunoadjuvants, and targeting ligands is ideal [1-8]. Additionally, due to their large surface area, nanoparticles can be readily surface-engineered with proteins, peptides, polymers, cell-penetrating moieties, reporter groups, and other functional and targeting ligands. The ease of design and use combined with multifunctionality makes using nanoparticles a versatile and useful delivery strategy for vaccines and immunotherapies.

In this special theme issue, we cover recent progress in the development and application of a variety of different classes of nanoparticles in immunotherapeutic applications.

\footnotetext{
${ }^{1}$ Department of Pharmaceutical Sciences and Experimental Therapeutics, College of Pharmacy, University of Iowa, Iowa City, IA 52242, USA.

${ }^{2}$ To whom correspondence should be addressed. (e-mail: aliasgersalem@uiowa.edu;)
}

These include peptide amphiphile micelles [9], $\mathrm{pH}$ responsive polymer-based nanoparticles [10], cationic liposomes [11], polysaccharide-based nanoparticles [12], and nanoparticles formed from biodegradable polymers such as polylactic-co-glycolide (PLGA) [13-15] and polyanhydrides [16]. This theme issue includes a comprehensive review by Jewell and colleagues on the topic of harnessing biomaterials to engineer the lymph node microenvironment for immunity or tolerance [17]. The focus on the lymph nodes is important because the lymph nodes and other secondary lymphoid organs, such as the spleen, play crucial roles in determining if and how immune responses develop following vaccination or immunotherapy. This theme issue also includes several original research articles. Tirrell and colleagues report on recent progress in the development of peptide amphiphile micelles that selfadjuvant group A streptococcal vaccinations [9]. In their report, a streptococcus B lymphocyte antigen and a dialkyl hydrophobic moiety were covalently linked and underwent self-assembly into micelles when added to water, due to the hydrophobic interactions among the alkyl tails. Upon vaccination of mice with these micelles, a potent IgG1 antibody response was induced that was similar to responses obtained from co-administration of soluble peptide with two classical adjuvants. Stayton and colleagues report on the enhancement of MHC-I antigen presentation via architectural control of $\mathrm{pH}$-responsive endosomolytic polymer nanoparticles [10]. In their study, increased MHC-I antigen presentation was observed in vitro with antigenloaded nanoparticles formulated from hyperbranched and cross-linked polymer structures compared to soluble antigen or antigen-loaded nanoparticles made from linear polymers. Narasimhan and colleagues report on the safety and biocompatibility of carbohydrate-functionalized polyanhydride nanoparticles [16]. In their study, carbohydrate-functionalized polyanhydride nanoparticles 
were shown to be safe when administered to mice via parenteral or intranasal routes. Jiskoot and colleagues report that cationic liposomes loaded with a synthetic long peptide (SLP) and the TLR3 ligand polyinosinic-polycytidylic acid (poly(I:C)) can act as a potent vaccine for induction of antigen-specific cytotoxic T lymphocytes (CTLs) [11]. In their experiments, the SLP, containing the model CTL epitope SIINFEKL, and poly(I:C) were co-loaded into cationic liposomes comprising DOTAP and DOPC. Studies demonstrated the capacity of these loaded liposomes to deliver their cargo effectively to DCs in vitro as well as trigger functional SLP-specific CTL responses in vivo. Weiner and colleagues report on the use of biodegradable microparticles (MPs) co-loaded with doxorubicin (Dox) and CpG ODN for in situ immunization against cancer [13]. The in situ immunization protocol implemented in these studies was founded on the principle that immune tolerance to a range of tumor antigens can be broken through the induction of immunogenic tumor cell death, promoted by Dox, which thereby provides DCs with abundant tumor antigen for presentation to, and subsequent activation of, tumor-specific CTLs. The presence of $\mathrm{CpG}$ is known to enhance antigen presentation by DCs and promote enhanced cellular immune responses, while PLGA MPs not only allow the safe co-delivery of their cargo, but they also abrogate the vesicant action of Dox. Using an in vivo B lymphoma model where mice were challenged on both left and right flanks with tumor, Weiner and colleagues demonstrated that intratumoral administration (on one flank) of Dox/CpG MPs combined with systemic codelivery of a negative checkpoint antagonist (anti-CTLA4 antibody) and a CD134 agonist resulted in efficient antitumor responses capable of eliminating both the treated and untreated (distal) tumors. Berkland and colleagues report on how structure, size, and solubility of antigen arrays determines efficacy in experimental autoimmune encephalomyelitis (EAE) [18]. They then show how co-delivery of autoantigen and B7 pathway modulators suppresses EAE [19]. Norian and colleagues report on a therapeutic vaccine involving tumor lysate-loaded MPs that reduce spontaneous metastases in a murine breast cancer model [15]. There is currently no cure for metastatic breast cancer, and available therapies are often associated with severe side effects. In Norian's studies, PLGA MPs injected subcutaneously were efficiently phagocytosed by DCs and localized to draining lymph nodes within $48 \mathrm{~h}$. A heterologous prime/boost vaccination therapy, where tumor lysate-loaded PLGA MPs were used as the prime and soluble tumor lysate plus a cocktail of adjuvants were used as the boost, resulted in a $42 \%$ reduction in metastatic lung tumor burdens compared to unvaccinated mice. It was also shown that this vaccine strategy did not induce autoimmunity. D'Souza and colleagues report on the induction of death receptor CD95 and co-stimulatory molecules $\mathrm{CD} 80$ and $\mathrm{CD} 86$ by meningococcal capsular polysaccharide-loaded vaccine nanoparticles [12]. Neisseria meningitidis is the primary cause of bacterial meningitis and sepsis worldwide, and virulence is mostly dependent on capsular polysaccharides (CPS) that define at least 12 serogroups and are an attractive target for protective vaccines. Although CPS-loaded albumin-based nanoparticles were found to induce expression of co-stimulatory molecules and acts as antigen depots, thereby sparing antigen dose, high doses of CPS lead to upregulation of CD95 resulting in decreased immunogenicity and decreased viability of antigen presenting cell populations. Finally, my research group reported on the development of a PLGA particle vaccine that can protect against house dust mite induced allergy [14]. Our study showed that the size of PLGA particles used for vaccination plays a major role in the prevention of house dust miteinduced allergy and that incorporation of $\mathrm{CpG}$ into the PLGA particles preferentially develops a Th1-type immune response.

Collectively, these studies provide examples of the recent and ongoing significant progress in the development of nanoparticle-based vaccines. This theme issue highlights the range of materials that can be used to prepare the nanoparticles and the many types of nanoparticles from micelles to liposomes that can be used to deliver vaccines. Pre-clinical studies presented in this theme issue show that nanoparticles can be safe and can be utilized for vaccine delivery to enhance therapeutic responses against a wide range of diseases from dust mite allergies to cancers. Nanoparticles have significant potential for further development in vaccine delivery and it is becoming increasingly clear that each disease will need nanoparticles with specific features that are tailored to enhancing the therapeutic response to that specific disease. For each vaccine formulation that is developed, the safety, route of administration [20], and formulation characteristics must be carefully considered prior to testing and use.

\section{REFERENCES}

1. Ahmed KK, Geary SM, Salem AK. Applying biodegradable particles to enhance cancer vaccine efficacy. Immunol Res. 2014;59(1-3):220-8.

2. Joshi VB, Geary SM, Salem AK. Biodegradable particles as vaccine antigen delivery systems for stimulating cellular immune responses. Hum Vaccin Immunother. 2013;9(12):2584-90.

3. Joshi VB, Geary SM, Salem AK. Biodegradable particles as vaccine delivery systems: size matters. AAPS J. 2013;15(1):8594.

4. Joshi VB, Geary SM, Carrillo-Conde BR, Narasimhan B, Salem AK. Characterizing the antitumor response in mice treated with antigen-loaded polyanhydride microparticles. Acta Biomater. 2013;9(3):5583-9.

5. Krishnamachari Y, Geary SM, Lemke CD, Salem AK. Nanoparticle delivery systems in cancer vaccines. Pharm Res. 2011;28(2):215-36.

6. Krishnamachari Y, Salem AK. Innovative strategies for codelivering antigens and $\mathrm{CpG}$ oligonucleotides. Adv Drug Deliv Rev. 2009;61(3):205-17. 
7. Zhang XQ, Dahle CE, Weiner GJ, Salem AK. A comparative study of the antigen-specific immune response induced by codelivery of $\mathrm{CpG}$ ODN and antigen using fusion molecules or biodegradable microparticles. J Pharm Sci. 2007;96(12):3283-92.

8. Zhang XQ, Dahle CE, Baman NK, Rich N, Weiner GJ, Salem AK. Potent antigen-specific immune responses stimulated by codelivery of $\mathrm{CpG}$ ODN and antigens in degradable microparticles. J Immunother. 2007;30(5):469-78.

9. Trent A, Ulery BD, Black MJ, Barrett JC, Liang S, Kostenko Y, David NA, Tirrell MV: Peptide amphiphile micelles self-adjuvant group A streptococcal vaccination. AAPS J, 2014.

10. Wilson JT, Postma A, Keller S, Convertine AJ, Moad G, Rizzardo E, Meagher L, Chiefari J, Stayton PS: Enhancement of MHC-I Antigen Presentation via Architectural Control of pH-Responsive, Endosomolytic Polymer Nanoparticles. AAPS J, 2014.

11. Varypataki EM, van der Maaden K, Bouwstra J, Ossendorp F, Jiskoot W. Cationic liposomes loaded with a synthetic long Peptide and poly(i:C): a defined adjuvanted vaccine for induction of antigen-specific T cell cytotoxicity. AAPS J. 2015;17(1):216-26.

12. Ubale RV, Gala RP, Zughaier SM, D'Souza MJ. Induction of death receptor CD95 and co-stimulatory molecules CD80 and CD86 by meningococcal capsular polysaccharide-loaded vaccine nanoparticles. AAPS J. 2014;16(5):986-93.

13. Makkouk A, Joshi VB, Wongrakpanich A, Lemke CD, Gross BP, Salem AK, et al. Biodegradable microparticles loaded with doxorubicin and $\mathrm{CpG}$ ODN for in situ immunization against cancer. AAPS J. 2015;17(1):184-93.
14. Joshi VB, Adamcakova-Dodd A, Jing X, Wongrakpanich A, Gibson-Corley KN, Thorne PS, et al. Development of a poly (lactic-co-glycolic acid) particle vaccine to protect against house dust mite induced allergy. AAPS J. 2014;16(5):975-85.

15. Gross BP, Wongrakpanich A, Francis MB, Salem AK, Norian LA. A therapeutic microparticle-based tumor lysate vaccine reduces spontaneous metastases in murine breast cancer. AAPS J. 2014;16(6):1194-203.

16. Vela-Ramirez JE, Goodman JT, Boggiatto PM, Roychoudhury R, Pohl NL, Hostetter JM, et al. Safety and biocompatibility of carbohydrate-functionalized polyanhydride nanoparticles. AAPS J. 2015;17(1):256-67.

17. Andorko JI, Hess KL, Jewell CM. Harnessing biomaterials to engineer the lymph node microenvironment for immunity or tolerance. AAPS J, 2014.

18. Sestak JO, Fakhari A, Badawi AH, Siahaan TJ, Berkland C. Structure, size, and solubility of antigen arrays determines efficacy in experimental autoimmune encephalomyelitis. AAPS J. 2014;16(6):1185-93.

19. Northrup L, Sestak JO, Sullivan BP, Thati S, Hartwell BL, Siahaan TJ, et al. Co-delivery of autoantigen and b7 pathway modulators suppresses experimental autoimmune encephalomyelitis. AAPS J. 2014;16(6):1204-13.

20. Murugappan S, Patil HP, Frijlink HW, Huckriede A, Hinrichs WL. Simplifying influenza vaccination during pandemics: sublingual priming and intramuscular boosting of immune responses with heterologous whole inactivated influenza vaccine. AAPS J. 2014;16(2):342-9. 